\title{
Sensitive periods and language aptitude in second language acquisition
}

\author{
MICHAEL H. LONG \\ University of Maryland \\ GISELA GRANENA \\ Universitat Oberta de Catalunya
}

(Received: January 21, 2018; final revision received: January 21, 2018; accepted: January 22, 2018; first published online 13 March 2018)

Keywords: critical period, sensitive periods, ultimate attainment, discontinuities, language aptitude

Mayberry and Kluender (Mayberry \& Kluender) adopt interesting positions on a wide range of issues. Due to space limitations, we will focus on just two where our views differ: (i) the robustness of maturational constraints on second language acquisition (L2A), and (ii) the implications of a role for language aptitudes within the boundaries defined by sensitive periods (SPs).

The distinction between CRITICAL and SENSITIVE periods is crucial. A critical period (CP) of heightened sensitivity to environmental stimuli required for some aspects of animal learning and development is typically short and precisely defined, often in terms of hours or days, not years. The ability or behavior concerned cannot be learned or developed to normal levels after the period closes, or in some cases, learned or developed at all; hence, CRITICAL period. For at least five reasons, CP is less appropriate for describing language acquisition.

First, achievements within biologically determined optimal periods of sensitivity for L2A are more variable. Nativelike attainment is possible for learners whose first meaningful L2 exposure (age of onset, or AO) in some domains differs by as much as two or three years. For instance, some children first exposed to a L2 at age 2, 3 or 4 can attain nativelike L2 phonology, and among learners first exposed as early as age 6 , and as late as the mid-teens, some do, and some do not, achieve nativelike command of L2 morphology and syntax.

Second, achievement of nativelike abilities in L2A cannot be explained in terms of a single CP. Different AOs predict ultimate attainment in different linguistic domains, with a series of offsets for phonology, lexis and collocations, and morphology and syntax, in that order (Granena \& Long, 2013).

Third, offsets are gradual, and less catastrophic in their effects. Very advanced (but not nativelike) L2 abilities are possible for a few individuals whose AO occurred well after the offset for the domain in question.
Fourth, L2 learners have already learned one or more languages, usually as children, a fact with some negative, but mostly positive, effects on subsequent language learning. Typological distance between L1 and L2 can affect rate and ultimate attainment, but always within the limits imposed by AO.

Fifth, humans differ in working memory and other aptitudes for implicit and explicit language learning. These differences affect rate of learning and ultimate L2 attainment, but cannot overcome biologically based maturational constraints (Granena, 2016).

Mayberry and Kluender speak of a single CP, from whose effects about $4 \%$ of post-CP adult starters are supposedly immune. They cite ostensible counterevidence in the form of individuals who have achieved native standards despite starting L2A after closure of some version of a CP. Detailed re-analyses (e.g., Long, 2005, 2013) of those studies and others have shown that apparent exceptions are the result of methodological limitations or flaws. Problems include one or more of confusion of early rate of acquisition and ultimate attainment, inappropriate choice of subjects, misleading operationalization of $\mathrm{AO}$ or AoA (age of arrival), biasing of results through use of leading instructions to raters, ceiling effects due to use of overly easy tests of L2 abilities, basing rater assessments of nativelikeness on very limited and/or rehearsed speech samples and/or "language-like behavior" (reading word lists aloud, etc.), differences between the variety of a language spoken by the non-natives and the native judges, juxtaposition of markedly non-native speech samples making near-native samples seem nativelike to raters, use of unreliable or invalid measures, inappropriate L1-L2 pairings, and faulty interpretations of statistical patterns.

Methodologically credible research requires screening of subjects into studies, use of multiple complex tasks and tests, including objective acoustic measures of accent

Address for correspondence:

Michael H. Long, Second Language Acquisition Program, School of Languages, Literatures, and Cultures, 3124 Jimenez Hall, University of Maryland, College Park, MD 20742, USA

mlong5@umd.edu 
or perception, rather than subjective ratings, alone, to preempt unwarranted classification of non-natives as native speakers, so-called "false positives" (Abrahamsson \& Hyltenstam, 2009).

Mayberry and Kluender assert that L2 proficiency declines with AoA, and that "results leave it mostly to the observer to decide whether the linearity vs. discontinuity glass is half full or half empty." In fact, it is well established that data plateau in some age groups, and, as shown in Granena and Long (2013), statistical techniques (multiple linear regression analyses and beta coefficients) can determine whether observed discontinuities (qualitative changes in the rate of decline) differ significantly from gradual linear declines, rendering impressionistic judgments unnecessary. Linear relationships for whole datasets are not incompatible with discontinuities and breakpoints within those datasets.

Mayberry and Kluender claim that the putative CPL applies to L1 learning, and that variable $\mathrm{L} 2$ achievement is a consequence of the prior L1, not age differences. Not so. Many studies compare child and adult L2 learners. Both groups have an L1. The key difference between them is $\mathrm{AO}$, which turns out to be decisive for the ability to achieve nativelike $\mathrm{L} 2$ abilities.

Mayberry and Kluender claim that effects for aptitude show that maturation is not in control. However, differences in implicit/explicit aptitudes are compatible with differences in the degree of availability of implicit learning mechanisms resulting from maturation. Language aptitudes can influence attainment, but only within the bounds set by maturational constraints, never by overriding those constraints.

\section{References}

Abrahamsson, N., \& Hyltenstam, K. (2009). Age of onset and nativelikeness in a second language: Listener perception versus linguistic scrutiny. Language Learning, 59, 249306.

Granena, G. (2016). Age differences, maturational constraints, and implicit and explicit L2 learning. Brill Research Perspectives in Multilingualism and Second Language Acquisition, 1, 9-44.

Granena, G., \& Long, M. H. (2013). Age of onset, length of residence, language aptitude, and ultimate L2 attainment in three linguistic domains. Second Language Research 29, 3, 311-343.

Long, M. H. (2005). Problems with supposed counter-evidence to the critical period hypothesis. International Review of Applied Linguistics 43, 287-317.

Long, M. H. (2013). Maturational constraints on child and adult SLA. In G. Granena, \& M. H. Long (eds.), Sensitive periods, language aptitude, and ultimate L2 attainment, pp. 3-41. Amsterdam and Philadelphia: John Benjamins.

Mayberry, R. I., \& Kluender, R. (2017). Rethinking the critical period for language: New insights into an old question from American Sign Language. Bilingualism: Language and Cognition doi:10.1017/S1366728917000724 